\title{
A Semantic and Knowledge-Based Approach for Handover Management
}

\author{
Fulvio Yesid Vivas ${ }^{1}\left(\mathbb{D}\right.$, Oscar Mauricio Caicedo ${ }^{1, *(\mathbb{D})}$ and Juan Carlos Nieves ${ }^{2}$ (D) \\ 1 Departamento de Telemática, Universidad del Cauca, Popayán, Cauca 190002, Colombia; \\ fyvivas@unicauca.edu.co \\ 2 Department of Computing Science, Umeå University, 90187 Umeå, Sweden; juan.carlos.nieves@umu.se \\ * Correspondence: omcaicedo@unicauca.edu.co; Tel.: +57-(2)-8209900 (ext. 2127)
}

Citation: Vivas, F.Y.; Caicedo, O.M.;

Nieves, J.C. A Semantic and

Knowledge-Based Approach for Handover Management. Sensors 2021,

21, 4234. https://doi.org/10.3390/ s21124234

Academic Editors: Ramón Agüero Calvo and Luis Francisco Díez

Received: 6 May 2021

Accepted: 11 June 2021

Published: 21 June 2021

Publisher's Note: MDPI stays neutral with regard to jurisdictional claims in published maps and institutional affiliations.

Copyright: (c) 2021 by the authors. Licensee MDPI, Basel, Switzerland. This article is an open access article distributed under the terms and conditions of the Creative Commons Attribution (CC BY) license (https:/ / creativecommons.org/licenses/by/ $4.0 /)$.

\begin{abstract}
Handover Management (HM) is pivotal for providing service continuity, enormous reliability and extreme-low latency, and meeting sky-high data rates, in wireless communications. Current HM approaches based on a single criterion may lead to unnecessary and frequent handovers due to a partial network view that is constrained to information about link quality. In turn, HM approaches based on multicriteria may present a failure of handovers and wrong network selection, decreasing the throughput and increasing the packet loss in the network. This paper proposes SIM-Know, an approach for improving HM. SIM-Know improves HM by including a Semantic Information Model (SIM) that enables context-aware and multicriteria handover decisions. SIM-Know also introduces a SIM-based distributed Knowledge Base Profile (KBP) that provides local and global intelligence to make contextual and proactive handover decisions. We evaluated SIM-Know in an emulated wireless network. When the end-user device moves at low and moderate speeds, the results show that our approach outperforms the Signal Strong First (SSF, single criterion approach) and behaves similarly to the Analytic Hierarchy Process combined with the Technique for Order Preferences by Similarity to the Ideal Solution (AHP-TOPSIS, multicriteria approach) regarding the number of handovers and the number of throughput drops. SSF outperforms SIM-Know and AHP-TOPSIS regarding the handover latency metric because SSF runs a straightforward process for making handover decisions. At high speeds, SIM-Know outperforms SSF and AHP-TOPSIS regarding the number of handovers and the number of throughput drops and, further, improves the throughput, delay, jitter, and packet loss in the network. Considering the obtained results, we conclude that SIM-Know is a practical and attractive solution for cognitive HM.
\end{abstract}

Keywords: semantic information model; knowledge base profile; handover management; wireless networks

\section{Introduction}

Handover Management (HM) is responsible for making network (dis)connection decisions in a timely manner [1,2]. In this sense, $\mathrm{HM}$ is pivotal for providing service continuity, enormous reliability and extreme-low latency, and meeting sky-high data rates, in current and upcoming wireless communications [3,4]. To achieve efficient HM challenges need to be face that are related to high handover rates and ping-pongs in dense communication environments, leading to an increase in both the data flow latency and the packet loss and, consequently, to a reduction of the network throughput $[5,6]$. Users moving at moderate-to-high speed require a seamless handover mechanism with few failures $[7,8]$.

In the networking literature, we find two types of approaches that address HM, namely, single criterion-based and multicriteria-based. Approaches based on a single criterion, such as the Signal Strong First (SSF), usually consider only the link quality in the end-user device for carrying out handovers. SSF compares the Received Signal Strength Indication (RSSI) of available networks and selects the network with the highest signal [9]. 
Single criterion-based approaches operate with a constrained network view that disregards contextual information, such as movement velocity and application requirements, leading to unnecessary and frequent handovers. These handover issues can decrease throughput, increase packet loss and even cause network service disruption [10-12]. The multicriteriabased approaches in [13-22] use RSSI and context information as criteria for ranking the available networks; the top-ranked network is selected by the end-user device for performing the connection process. These approaches disregard one or more relevant criteria, such as wireless network characteristics (e.g., coverage area), user device features (e.g., battery consumption), application requirements (e.g., real-time response) or user peculiarities (e.g., mobility pattern), leading to the failure of handovers and wrong network selection, negatively impacting the network's performance [12,23]. Hybrid solutions combine multicriteria approaches [24]; however, their computational complexity is high.

This paper presents SIM-Know, an approach for improving HM. The contributions of SIM-Know are two-fold. SIM-Know proposes a Semantic Information Model (SIM) that allows us to make context-aware handover decisions by considering and relating criteria from several context information domains: Network, Application, User, UserDevice, and Handover. SIM-Know also introduces a SIM-based distributed Knowledge Base Profile (KBP) that offers local and global intelligence for making contextual and proactive decisions during the handover process. We evaluated SIM-Know in an emulated wireless network. When the end-user device moves at low and moderate speeds, the results show that our approach outperforms SSF (a single criterion approach) and behaves similarly to the Analytic Hierarchy Process combined with the Technique for Order Preferences by Similarity to the Ideal Solution (AHP-TOPSIS, a multicriteria approach), regarding the number of handovers and the number of throughput drops. SSF outperforms SIM-Know and AHP-TOPSIS regarding the handover latency metric because SSF runs a straightforward process for making handover decisions. At high speeds, SIM-Know outperforms SSF and AHP-TOPSIS regarding the number of handovers and the number of throughput drops and, further, improves the throughput, delay, jitter and packet loss in the network. From the obtained results, we conclude that SIM-Know is an attractive and feasible solution for cognitive HM.

The rest of this paper is organized as follows: Section 2 reviews the related work. Section 3 introduces SIM-Know, including SIM and KBP. Section 4 presents the evaluation of SIM-Know. Finally, some conclusions and future work are presented in Section 5.

\section{Related Work}

This section presents research on HM approaches based on both a single criterion and on multiple criteria. Table 1 briefly summarises each approach and presents the type of data/information model employed and how they make handover decisions. According to [25], the handover control can be: Network-Controlled HandOver (NCHO), MobileControlled HandOver (MCHO), Mobile-Assisted HandOver (MAHO) and Network-Assisted HandOver (NAHO). In NCHO, the network starts and controls the handover; operators usually adopt it for load balancing and traffic management. In MCHO-traditionally used by IEEE 802.11 technologies - the end-user device initiates and controls the handover. In $\mathrm{MAHO}$, the end-user device helps in the handover process controlled by the network; MAHO is typically used in cellular networks. In NAHO, the network helps with the handover process controlled by the end-user device; NAHO is intended for heterogeneous wireless networks.

Next, we point out the main shortcomings of the related work. The work in [9] employed SSF to make handover decisions. Since it does not exploit contextual information, such as movement velocity and application requirements for enhancing HM, SSF can lead to unnecessary and frequent handovers, decreasing throughput, increasing packet loss and even causing network service disruption. In [13-22] handover decisions are made by using multicriteria and techniques such as Fuzzy Logic, Network Management Policies or Multiple Attribute Decision Making (MADM) Algorithms. Nonetheless, these approaches 
neglect an information model that disregards criteria from one or more information domains, such as network characteristics and status, application requirements, end-user profile, end-user device features, or handover history, which are relevant for advancing HM. Thus, they can also lead to handover issues (generating failure, unnecessary and frequent handovers) and, consequently, network performance degradation. The work in [24] presents a solution that combines multicriteria approaches for improving HM. Nevertheless, its computational complexity is higher than that of the unique criterion and individual multicriteria methods. Unlike the related work, SIM-Know introduces an SIM to enable context-aware handover decisions by considering and relating criteria from a comprehensive set of context information domains: Network, Application, User, UserDevice and Handover. SIM-Know also proposes KBP, to realize cognitive and proactive handover decisions by providing local and global knowledge.

Table 1. Related Work.

\begin{tabular}{|c|c|c|c|c|}
\hline \multirow{2}{*}{ Work } & \multirow{2}{*}{ Description } & \multicolumn{2}{|c|}{ Making-Decision } & \multirow{2}{*}{ Wireless Technology } \\
\hline & & Control & Method & \\
\hline [9] & $\begin{array}{l}\text { The end-user device switches to another Access } \\
\text { Point (AP) or Base Station (BS) when the RSSI } \\
\text { level of the serving network is lower than } \\
\text { a threshold }\end{array}$ & $\mathrm{MCHO}$ & Policy & $\begin{array}{l}\text { Universal Mobile } \\
\text { Telecommunications } \\
\text { System, Wireless Local } \\
\text { Area Network (WLAN) }\end{array}$ \\
\hline [13] & $\begin{array}{l}\text { A Software-Defined Networking (SDN) } \\
\text { controller uses a fuzzy system to score } \\
\text { candidate networks for staying in the current } \\
\text { network or connecting to a better one }\end{array}$ & $\mathrm{NCHO}$ & Fuzzy Logic & Fifth Generation (5G) \\
\hline [14] & $\begin{array}{l}\text { An algorithm is proposed to reduce the } \\
\text { handovers by multicriteria decision-making } \\
\text { algorithms improved with a context-aware and } \\
\text { threshold-based scheme }\end{array}$ & $\mathrm{MCHO}$ & $\begin{array}{l}\text { TOPSIS, } \\
\text { PROMETHEE, } \\
\text { SAW }\end{array}$ & $\begin{array}{l}\text { 5G, Long-Term Evolution } \\
\text { (LTE), WLAN }\end{array}$ \\
\hline [15] & $\begin{array}{l}\text { A framework, based on a quantum-inspired } \\
\text { immune clonal algorithm and network-related } \\
\text { and user-related parameters with different } \\
\text { weights, is proposed for improving the access } \\
\text { network selection in wireless networks }\end{array}$ & $\mathrm{NCHO}$ & $\begin{array}{l}\text { Quantum } \\
\text { Computing }\end{array}$ & LTE, WLAN \\
\hline [16] & $\begin{array}{l}\text { A fuzzy logic and reinforcement learning-based } \\
\text { mechanism is introduced to address } \\
\text { unnecessary and frequent handovers by } \\
\text { adjusting the handover hysteresis margin and } \\
\text { time-to-trigger }\end{array}$ & $\mathrm{NCHO}$ & $\begin{array}{c}\text { Fuzzy } \\
\text { Q-Learning }\end{array}$ & LTE \\
\hline [17] & $\begin{array}{l}\text { A solution, based on SDN, Binary Integer Linear } \\
\text { Programming (BILP), user criteria and network } \\
\text { packet error rate data, is proposed to rank } \\
\text { candidate BSs and to enhance the handover } \\
\text { selection phase }\end{array}$ & $\mathrm{NCHO}$ & BILP & LTE \\
\hline
\end{tabular}


Table 1. Cont.

\begin{tabular}{|c|c|c|c|c|}
\hline \multirow{2}{*}{ Work } & \multirow{2}{*}{ Description } & \multicolumn{2}{|c|}{ Making-Decision } & \multirow{2}{*}{ Wireless Technology } \\
\hline & & Control & Method & \\
\hline [18] & $\begin{array}{l}\text { A framework, based on data analytics, context } \\
\text { extraction, user profiling and pre-processing } \\
\text { contextual information, is presented to score the } \\
\text { available BSs and to improve network access selection }\end{array}$ & NAHO & Fuzzy Logic & $5 G$ \\
\hline [19] & $\begin{array}{l}\text { The AP or BS selection is improved by using AHP for } \\
\text { weighting selection criteria coming from the user and } \\
\text { networks' context and TOPSIS for ranking the } \\
\text { available networks }\end{array}$ & $\mathrm{NCHO}$ & AHP-TOPSIS & LTE, WLAN \\
\hline [20] & $\begin{array}{l}\text { A mechanism is proposed for selecting the radio } \\
\text { access network that best meets the end-user needs by } \\
\text { considering the on/off state and battery level of the } \\
\text { mobile device and the available bandwidth in the } \\
\text { target and serving network }\end{array}$ & $\mathrm{MCHO}$ & Policy & LTE, WLAN \\
\hline$[21]$ & $\begin{array}{l}\text { A versatile modeling methodology is introduced for } \\
\text { evaluating proactive and reactive vertical handover } \\
\text { approaches }\end{array}$ & $\mathrm{NCHO}$ & Policy & 5G, LTE, WLAN \\
\hline [22] & $\begin{array}{l}\text { Two co-operating algorithms with adaptive } \\
\text { thresholds are introduced for performing network } \\
\text { selection while avoiding network congestion and } \\
\text { meeting user preferences regarding monetary cost, } \\
\text { Quality of Service (QoS), security and energy } \\
\text { consumption }\end{array}$ & NAHO & Policy & $5 \mathrm{G}$ \\
\hline [24] & $\begin{array}{l}\text { A multiattribute decision handover making scheme, } \\
\text { centered in the triggering phase and based on SDN } \\
\text { and Fuzzy Logic, is proposed for increasing the } \\
\text { network throughput and reducing unnecessary } \\
\text { handovers and total handover delay in femto-access } \\
\text { points and device-to-device communications }\end{array}$ & NAHO & $\begin{array}{l}\text { TOPSIS, } \\
\text { Fuzzy Logic, } \\
\text { AHP }\end{array}$ & LTE, WLAN \\
\hline
\end{tabular}

PROMETHEE: Preference ranking organization method for enrichment evaluation-SAW: Simple Additive Weighting Algorithm.

\section{SIM-Know}

HM allows an end-user device to keep an active connection when moving from one network's coverage area (BS or AP coverage) to another [9]. HM comprises the initiation, selection and execution phases $[17,26,27]$. Handover Initiation gathers all the information needed to identify and determine the neighboring networks and their current and future statuses (e.g., data about network performance and available services). Network Selection chooses the best available network from a ranking created by taking into account a single criterion or multiple criteria. Handover Execution connects and disconnects end-users to and from a network, involving resource allocation and releasing [28].

SIM-Know introduces SIM and KBP for improving HM. SIM allows SIM-Know to make context-aware handover decisions. In turn, the distributed KBP provides local and global intelligence to make rule-based cognitive decisions about network connection and disconnection. SIM and KBP envision diminishing the number of handovers and the number of throughput drops and, as a consequence, have a positive impact on several network performance metrics (delay, jitter, packet loss, and throughput). 


\subsection{Semantic Information Model}

SIM-Know makes appropriate and contextual handover decisions by considering criteria from several information domains (i.e., Network, Application, User, UserDevice, and Handover) modeled by SIM. We use the Common Information Model (CIM) and the Web Ontology Language (OWL) to carry out SIM (see Figure 1). We adopted CIM [29] because it provides high expressiveness for modeling, for management purposes, information systems, applications, and networks [30]. We used OWL [31] because it enables reasoning in the model and the sharing of knowledge among software agents [32]. In particular, SIM uses OWL classes and properties to characterize HM entirely by modeling the information domains and their relationships.

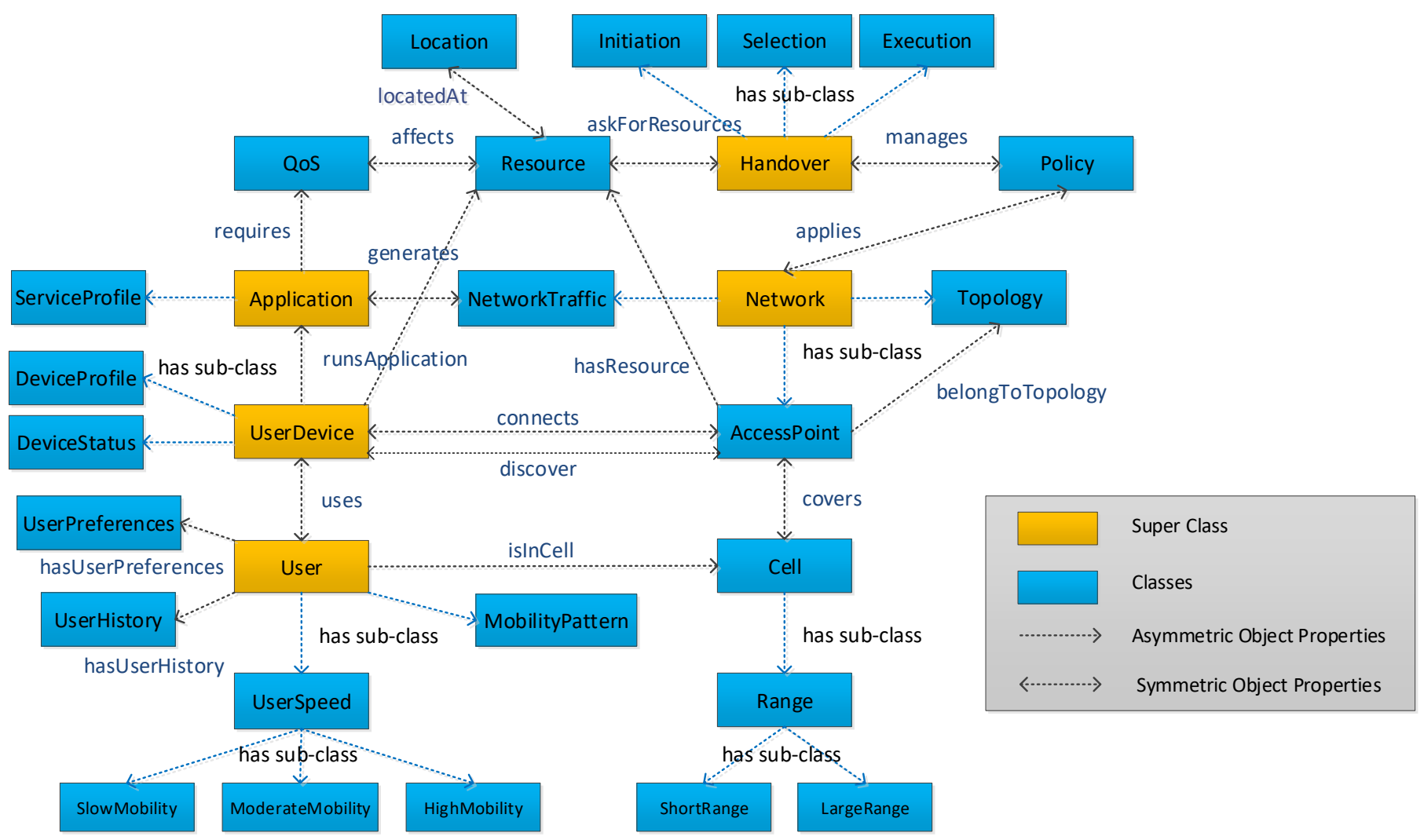

Figure 1. Semantic Information Model.

Figure 1 shows the five knowledge domains comprising SIM as superclasses, namely, Network, User, Application, UserDevice and Handover. It is worth noting that the Handover superclass models HM by using the Initiation, Selection, Execution and Policy classes. The other superclasses represent the information domains containing the parameters used to improve decision making in HM. Each superclass relates to other classes by the has sub-class relationship. The Initiation class models the initiation phase that defines when the selection phase is triggered, which, in turn, is modeled by the Selection class that is responsible for obtaining the candidate networks for performing the handover. The Execution class performs the handover itself, since it allocates and releases AP (i.e., AccessPoint class) and user device (i.e., UserDevice class) resources, represented by the Resource class, which affects the QoS required (i.e., QoS class) by a user application (i.e., Application class). The Policy class represents the policies to apply to the Network class and governs the HM process. An example of a policy is to rank the candidate networks considering some criteria such as users' speeds and their movement patterns.

The Network superclass models the characteristics and status of a network by using the Topology, NetworkTraffic, and AccessPoint classes. The Topology class represents the network's organization, including nodes and links. The NetworkTraffic class represents data 
and control traffic passing by the network. The AccessPoint class models a networking device using wireless technology; this class considers the area covered by the Cell class, which includes the Range class, which contains the LargeRange and SmallRange classes. The isCoveredByCell, hasResource and belongToTopology properties represent the AccessPoint class's relationship with the Cell, Resource and Topology classes, respectively. The Resource class models the ability to manage the resource consumption of APs located at (Location class) a particular network point.

The User superclass models the profile and behavior of the end-users by the UserPreferences, UserHistory, MobilityPattern and UserSpeed classes. The UserSpeed class includes the SlowMobility, ModerateMobility and HighMobility classes in order to model how fast an end-user moves. The UserPreferences class profiles the users with information related to, for instance, network preference by cost and service quality expectation. The UserHistory class models the historical (dis)connection of end-users. The MobilityPattern contains information about the end-users' mobility pattern, which is predictable from their trajectory and velocity. The Application superclass represents end-user applications with the ServiceProfile and QoS. The ServiceProfile class models the application's types (e.g., remote surgery, augmented reality, high definition video conferences). The QoS class allows the representation of a set of QoS requirements (e.g., delay, throughput and packet loss) for each type of application.

The UserDevice superclass models the end-user devices and their components by the DeviceProfile, DeviceStatus and Resource classes. The DeviceProfile class models the device's characteristics. The DeviceStatus class represents the device's current status (e.g., lowbattery and off-air). The Resource class models the ability to manage the resource consumption of end-user devices located at (Location class) a particular network point. The UserDevice superclass relates to the Application superclass via the runsApplication property, which allows knowledge of the applications that are running in each end-user device. The is UsedByUser property defines a relationship between UserDevice and User.

\subsection{Knowledge Base Profile}

KBP is a distributed knowledge base that intends to provide local and global intelligence that supports making rule-based cognitive decisions about network connection and disconnection processes. Figure 2 depicts the KBP internal structure, which is comprised of layers and processes. The Semantic layer uses SIM (the entire model or a part) to obtain information from the data included in the Context layer. The Reasoning layer obtains knowledge from the information represented by SIM. The Adaption process acts on the layers to maintain updated data, information and knowledge. The Collaboration process enables the sharing of the obtained knowledge between KBP instances. Next, we detail the KBP's layers and processes.

\subsubsection{Layers}

The Context layer includes contextual data about the user, network, device, application, and handover. Contextual data are essential for carrying out HM in environments with multiple wireless networks [20]. As in [21,33], this layer is divided into static and dynamic sublayers. The Static Context sublayer involves data that do not change or rarely do; it plays a vital role in assisting with neighbor network discovery [34]. Examples of static data include the wireless technology supported by the end-user devices and APs, and the wireless network technology coverage area. The Dynamic Context sublayer serves an updated network view, including dynamic data such as the application requirements of a device needing handover and capacity available in a target AP, which enables the upper layers (Semantic and Reasoning layers) to realize knowledge-based handovers.

The Semantic layer offers a SIM instance that is nourished by the bottom layer's contextual data. Thus, the Semantic layer structures the information to achieve intelligent, timely and context-aware HM (considering criteria from the Static and Dynamic contexts). For instance, the DeviceProfile, UserPreferences, UserSpeed and MobilityPattern SIM classes can 
be used to build up a map of candidate networks; overall, SIM classes provide a structure to contextual data. It is worth noting that we consider three KBP flavors depending on how they instantiate SIM. $K B P_{N}$, located at any AP or BS, instantiates the superclasses Network and Handover. $K B P_{M}$, located at end-user devices, instantiates the superclasses Application, User, UserDevice and Handover. $K B P_{S}$ is a complete KBP that can run on a logically centralised entity (e.g., a controller in a software-defined wireless network). The SIM's distribution allows any KBP (SIM-Know) to create and share local knowledge to generate global knowledge.

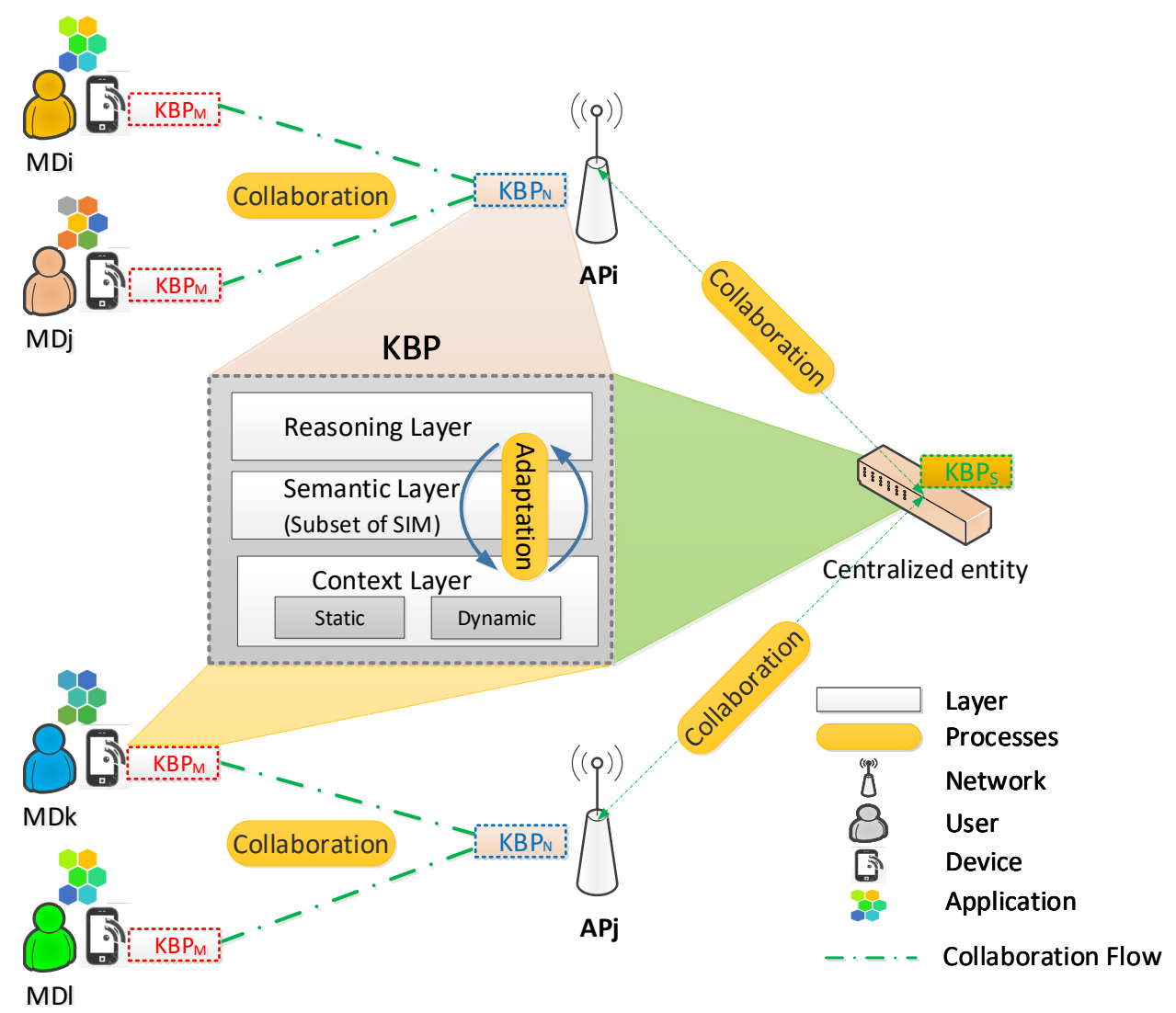

Figure 2. Knowledge Base Profile.

The Reasoning layer triggers the Initiation phase, selects the target network and realizes the handover itself by inferring knowledge from SIM. We use Description Logic (DL) [35] to express in a structured and formal way the rules governing the Reasoning layer and, so, HM; the reasoning rules generate local and global intelligence to make autonomous handover connection decisions. Each rule has a set of conditions and settings. To illustrate how the Reasoning layer operates, next, we present some of the rules that are modeled to realize a policy intended to select candidate networks proactively, considering the coverage of APs and the mobility pattern of end-user devices. For example, the Rule APInRange (see Listing 1) serves to discover neighboring networks considering RSSI.

Listing 1. Rule for APInRange.

APInRange $\equiv$ User $\sqcap \exists$ isInCell.( $\exists$ covers.AccessPoint)

Listing 2 shows that the Rule UserSpeed is useful for defining the speed of users. If a User is moving with $v>t h_{u}$, he/she has a UserHighSpeed. If a User is moving with a speed higher than $t h_{l}$ and lower than or equal to $t h_{u}$, he/ she has a UserModSpeed. UserSlowSpeed is when the user moves with $v \leq t h_{l}$. According to [36], $t h_{u}$ can be set to $50 \mathrm{Km} / \mathrm{h}$ and $t h_{l}$ to $10 \mathrm{Km} / \mathrm{h}$. 
Listing 2. Rule for UserSpeed.

UserHighSpeed $\equiv$ User $\sqcap$ ( $\exists$ hasUserSpeed.HighMobility)

UserModSpeed $\equiv$ User $\sqcap$ ( $\exists$ hasUserSpeed.ModerateMobility)

UserSlowSpeed $\equiv$ User $\sqcap(\exists$ hasUserSpeed.SlowMobility $)$

Listing 3 shows that Rule APRange is helpful for listing the APs by coverage range. LargeRange is given by range $>t h_{r}$ and ShortRange by range $\leq t h_{r}$. According to [14], $t h_{r}$ can be set to $35 \mathrm{~m}$ for 802.11ac. Listing 4 presents Rule SoJournTime, which is useful for determining the time the user stays covered by an AP. If UserSlowSpeed is moving in APLRange, it results in LongSojournTime. If UserHighSpeed is moving in APSRange, it results in SmallSojournTime. A MSojournTime happens when UserHighSpeed or UserModSpeed is moving in APLRange. If UserSlowSpeed or UserModSpeed is moving in APSrange, it also results in MSojournTime. SmallSojournTime may be more challenging than LongSojournTime and MSojournTime in 5G networks and beyond is characterized by small coverage areas and high-mobility.

Listing 3. Rule for APRange.

APLRange $\equiv A P \sqcap \exists$ isCoveredBy.( $\exists$ hasRange.LargeRange)

APSRange $\equiv A P \sqcap \exists$ isCoveredBy.( $\exists$ hasRange.ShortRange)

Listing 4. Rule for SoJournTime.

LongSojournTime $\equiv$ UserSlowSpeed $\sqcap(\exists$ APLRange $)$

SmallSojournTime $\equiv$ UserHighSpeed $\sqcap(\exists$ APSRange $)$

MSojournTime $\equiv$ APLRange $\sqcap($ UserHighSpeed $\sqcup$ UserModSpeed $) \sqcup$ APSRange $\sqcap$ (UserSlowSpeed $\sqcup$ UserModSpeed)

Listing 5 shows that Rule Candidate AP is useful for creating the list of candidate APs for end-user devices with MSojournTime or LongSojournTime in the network. Listing 6 presents Rule Associate AP, which links the end-user device with the first AP in the list of candidates. It is worth noting that each network administrator can define his/her own rules to manage the wireless network as he/she needs.

Listing 5. Rule for CandidateAP.

Candidate AP $\equiv$ MSojournTime $\sqcup$ LongSojournTime

Listing 6. Rule for AssociateAP.

AssociationToAP $\equiv$ User $\sqcap \exists$ Uses.UserDevice( $\exists$ Connects.AP)

\subsubsection{Processes}

The Adaptation process allows SIM-Know to dynamically adapt to the changing environments and to enhance HM by modifying the content of the layers of the KBP instances. The content is modified in a bottom-up way, starting with the contextual data, followed by the SIM instances, and ending with the acquired knowledge when environmental changes happen, such as new networks appearing, dynamic traffic conditions and variations in QoS requirements. Furthermore, this process allows the addition and updating of the Reasoning layer seeking to meet QoS and to preserve network performance.

The Collaboration process allows $\mathrm{KBP}\left(K B P_{N}, K B P_{S}, K B P_{M}\right.$ and any other profile defined to extend SIM-Know) to interchange the knowledge obtained for enhancing the decision-making in HM. For instance, the collaboration between $K B P_{M}$ and $K B P_{N}$ would allow for choosing the optimal and appropriate time to trigger the handover and select the most suitable access network according to the end-user QoS requirements and network status. 


\subsection{Operation}

Figure 3 presents how SIM-Know operates in WLAN. First, $K B P_{S}, K B P_{M}$ and $K B P_{N}$ collect their Static Context. Second, $K B P_{M}$ monitors and updates the Dynamic Context information related to, for instance, end-user speed, APs in range and RSSI. In parallel, $K B P_{M}$ requests from $K B P_{N}$ the Dynamic Context information, which includes the associated and in range end-user devices. Third, based on the Static Context and Dynamic Context, every KBP generates its local knowledge. For example, the local knowledge in $K B P_{M}$ can be HighMobility and in $K B P_{N}$ can be LargeRange. Fourth, $K B P_{M}$ launches the handover process. Fifth, the Collaboration process starts between the corresponding $K B P_{M}$ and $K B P_{N}$ and ends with sending knowledge to $K B P_{S}$. Sixth, $K B P_{S}$ builds up the global network view, generates a handover policy for selecting candidate APs according to the rules defined in its Reasoning Layer, and sends those candidates to $K B P_{M}$. Seventh, $K B P_{M}$ selects the Target $K B P_{N}$ by using the rules defined in its Reasoning Layer. Eighth, $K B P_{M}$ sends a Handover Request to the Target $K B P_{N}$, which sends back an acknowledgment to $K B P_{M}$. Ninth, $K B P_{M}$ sends disconnection requests to the current Serving $K B P_{N}$, which, in turn, sends a disconnection acknowledgment to $K B P_{M}$. Tenth, every $K B P$ executes the Adaptation process to handle the context variations dynamically.
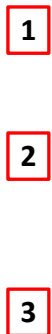

4

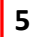

6

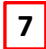

8

5
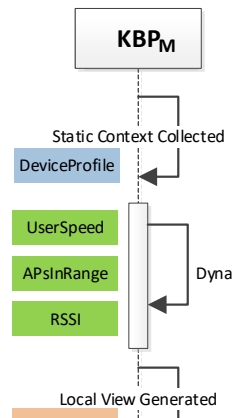

(1)
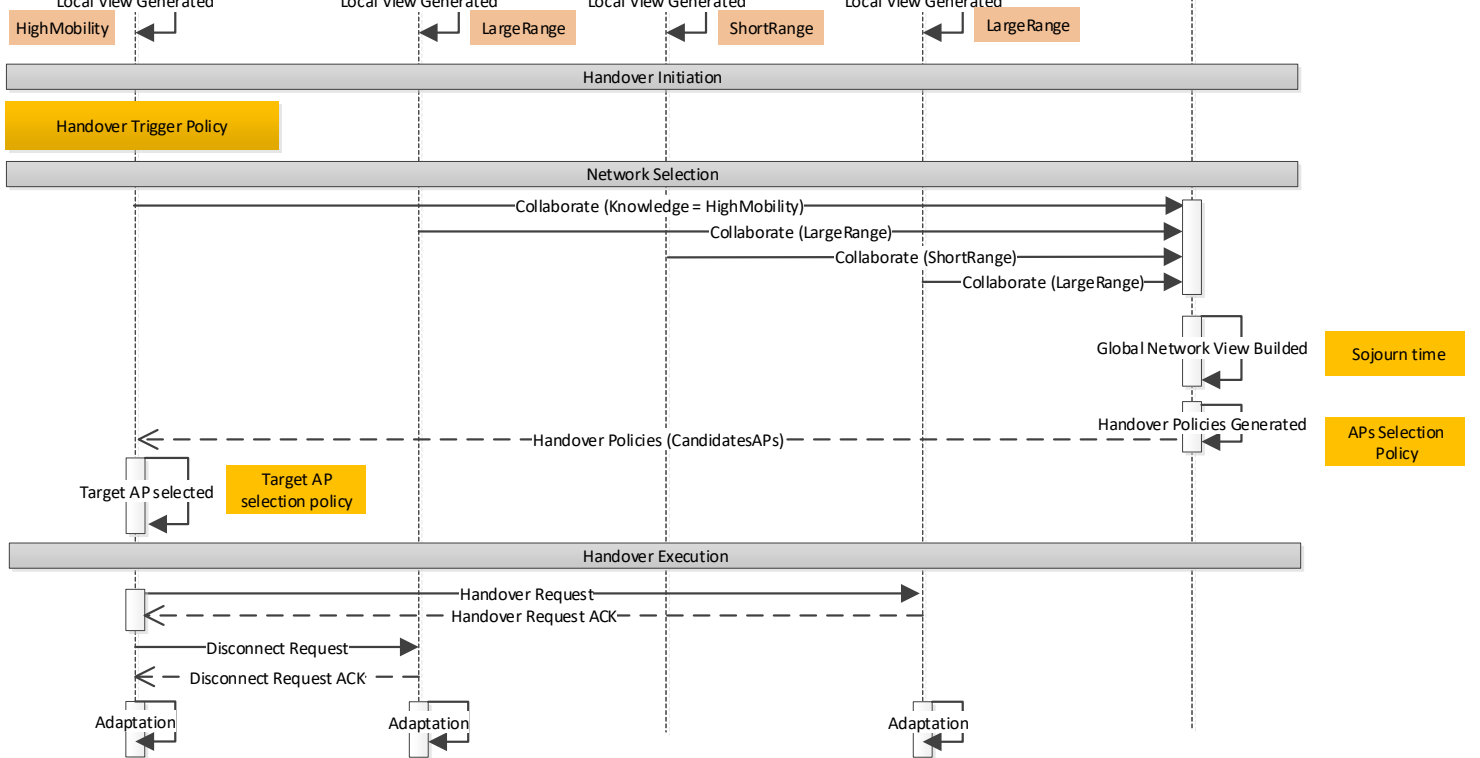

Figure 3. SIM-Know Operation.

Figure 4 shows the data format used in $K B P_{S}$ to store the global knowledge built with the information coming from $K B P_{N}$ and $K B P_{M}$. The format follows the triplet (Subject, Predicate, Object) encoded in Entity Notation [37], which enables a lightweight knowledge representation for resource-constrained environments. The Subject identifies a class in SIM by the combination of ClassId (e.g., ceu101) and ClassType (e.g., User). Each Subject is related to various pairs, Predicate-Object. The Predicate identifies a property (e.g., UserSpeed) of the Subject while the Object provides the value of such a property 
(e.g., HighMobility). The Object can also be a ClassId, to represent relationships among Subjects.

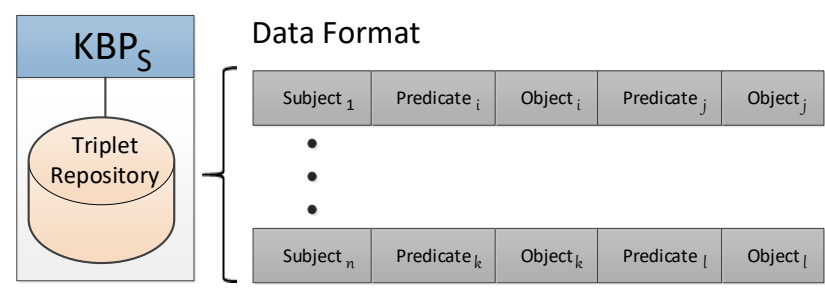

Figure 4. $K B P_{S}$ Data Format.

Figure 5 illustrates how SIM-Know can operate in $5 \mathrm{G}$ by running $K B P_{M}$ in the User Equipment (UE), $K B P_{N}$ in gNodeB $(\mathrm{gNB})$, and $K B P_{S}$ in the Core Network $(\mathrm{CN})$. The handover in 5G, according to the specification 3GPP TS38.300 [38], consists of three phases: preparation (steps 0-5), execution (steps 6-8), and completion (steps 9-12).

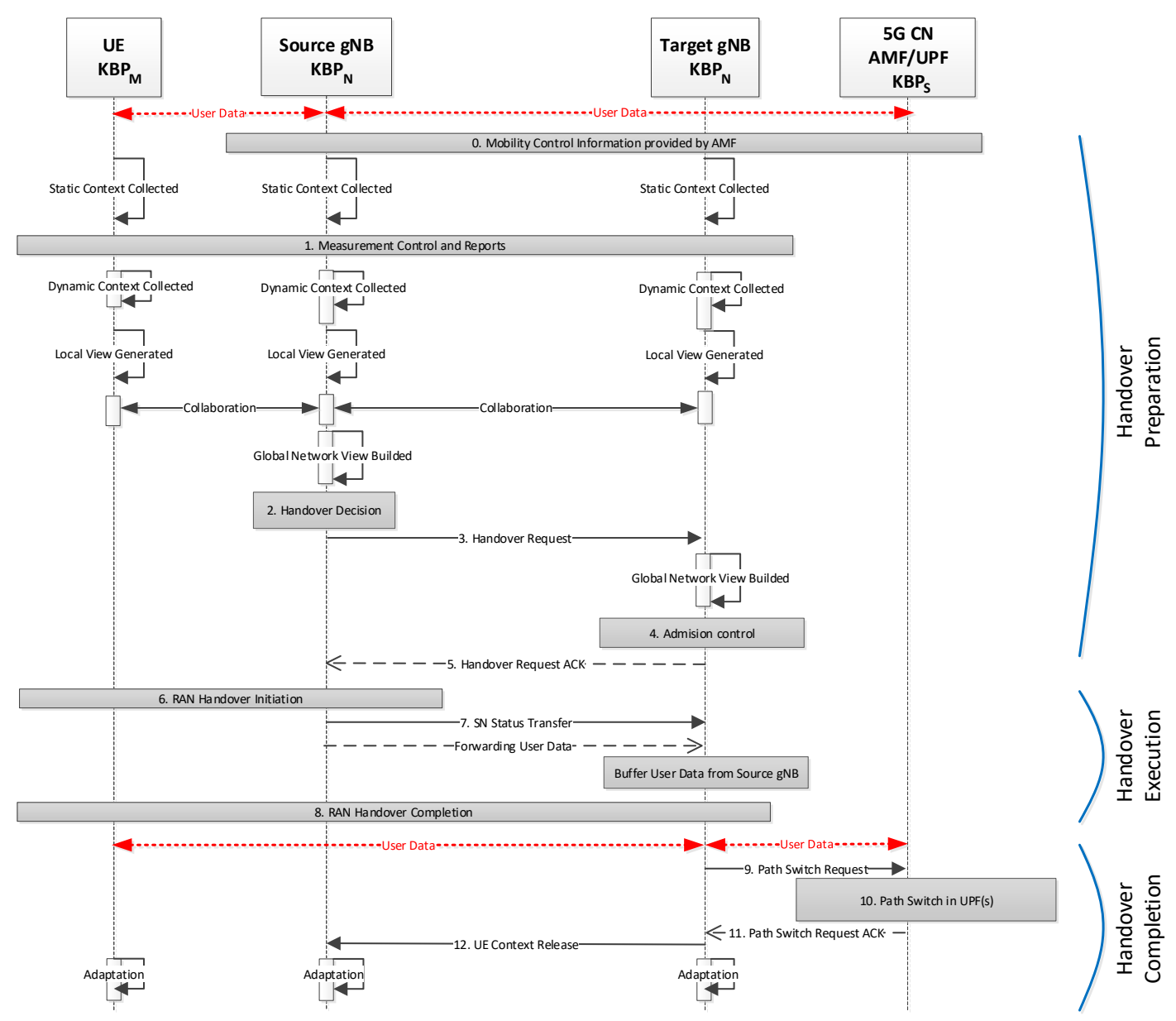

Figure 5. SIM-Know in 5G Intra-AMF/UPF Handover.

The steps are as follows:

- $\quad$ Step 0: Each KBP gathers the Static context.

- Step 1: $K B P_{M}$ and $K B P_{N}$ initialize measuring procedures to collect the Dynamic context, generate local knowledge, and exchange Measurement Reports by way of the Collaboration process. Source $K B P_{N}$ builds up its local knowledge.

- $\quad$ Step 2: Source $K B P_{N}$, based on its local knowledge, makes handover decisions. gNBs are responsible for making handover decisions. 
- $\quad$ Step 3: Source $K B P_{N}$ sends a handover request message to Target $K B P_{N}$.

- Step 4: Target $K B P_{N}$ executes the admission control procedure based on its local knowledge.

- Step 5: Target $K B P_{N}$ sends a handover request acknowledgment to Source $K B P_{N}$.

- Step 6: Source $K B P_{N}$ sends a handover command to $K B P_{M}$ for handover initiation.

- $\quad$ Step 7: Source $K B P_{N}$ sends the sequence number status transfer message to Target $K B P_{N}$. Source $K B P_{N}$ may initiate data forwarding.

- Step 8: $K B P_{M}$ detaches from Source $K B P_{N}$ and synchronizes with Target $K B P_{N}$.

- Step 9: Target $K B P_{N}$ informs $K B P_{S}$ that $K B P_{M}$ has changed the cell by way of the path switch request message.

- $\quad$ Step 10: $K B P_{S}$ switches the data path towards Target $K B P_{N}$.

- Step 11: $K B P_{S}$ acknowledges the path switch request message.

- Step 12: Target $K B P_{N}$ informs Source $K B P_{N}$ that the handover was successful and triggers the release of resources for Source $K B P_{N}$ by sending a UE Context Release message. Finally, Source $K B P_{N}$ releases the resources associated with $K B P_{M}$, invoking the Adaptation process.

\section{Evaluation}

This section presents the evaluation of SIM-Know in a WLAN, aiming to show its behavior regarding the number of handovers and the number of throughput drops, and its impact on various typical network performance metrics. Section 4.1 depicts the SIMKnow's prototype and the test environment. Section 4.2 shows the performance metrics and traffic generation. Section 4.3 presents and discusses the results of SIM-Know and two well-known handover solutions.

\subsection{Prototype and Test Environment}

We implemented the SIM-Know prototype for WLAN, including $K B P_{M}, K B P_{N}$, and $K B P_{S}$, by using the Python programming language version 2.7 . We also deployed the prototype in a Mininet-WiFi emulator [39] (see Figure 6) running on an Ubuntu 16.04 Virtual Machine (VM) with a Core i7-3630 processor and 8 GB RAM. Mininet-WiFi adds virtual BSs and APs to classical Mininet [40] to enable the emulation of wireless network environments. The SIM-Know prototype, as well as all test scripts, are available in the project repository [41].

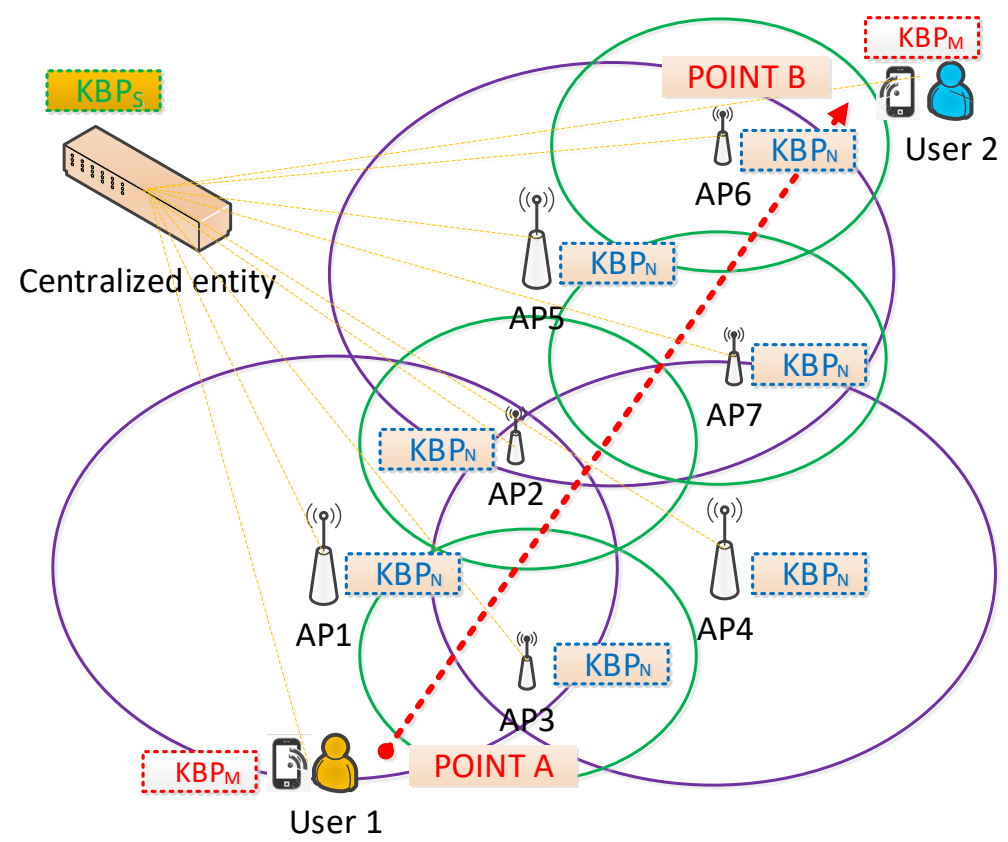

Figure 6. Test Environment. 
Figure 6 shows the WLAN test scenario in which we evaluated and compared SIMKnow, SSF, and AHP-TOPSIS. The scenario, deployed in Mininet-WiFi, included seven APs, an end-user device associated with User1, and another end-user device linked to User2. In particular, we used three APs with a large coverage range (i.e., up to $75 \mathrm{~m}$ for $A P_{1}, A P_{4}$, and $A P_{5}$ with $802.11 n$ ) and four with a short coverage range (i.e., up to $35 \mathrm{~m}$ for $A P_{2}, A P_{3}, A P_{6}$, and $A P_{7}$ with 802.11ac). We also analyzed the performance when the User 1 moved from Point A to Point B by following a straight line without directional change at a constant speed. We used three speeds for testing: $1.42 \mathrm{~m} / \mathrm{s}$ corresponding to SlowMobility, $3.74 \mathrm{~m} / \mathrm{s}$ to ModerateMobility, and $13.41 \mathrm{~m} / \mathrm{s}$ to HighMobility. The end-user device associated with User 1 transmitted traffic (Voice over IP (VoIP) or Transmission Control Protocol (TCP)) to the end-user device linked to User 2 , which was immobile. We repeated the experiments thirty-three times to obtain results with a $95 \%$ confidence level. Table 2 summarizes the setup of the experiments.

Table 2. Experiment Setup.

\begin{tabular}{cc}
\hline Parameters & Value \\
\hline Wireless technology & $802.11 \mathrm{n}, 802.11 \mathrm{ac}$ \\
\hline Emulation area & $180 \times 180 \mathrm{~m}$ \\
\hline Carrier frequency & $2 \mathrm{GHz}$ \\
\hline Channel bandwidth & $20 \mathrm{MHz}$ \\
\hline $\begin{array}{c}\text { Transmission power of cells } \\
\text { large-range/short-range }\end{array}$ & $25 / 14 \mathrm{dBm}$ \\
\hline Path loss model from cells & Log-Distance Propagation Loss/ITU-R P1283 \\
\hline Emulation time for HighMobility & $30 \mathrm{~s}$ \\
\hline Emulation time for ModerateMobility & $180 \mathrm{~s}$ \\
\hline Emulation time for SlowMobility & $\begin{array}{c}\text { Flows with constant inter-departure time between packets } \\
(1000 \text { pkts/s) and constant packets size (512 bytes) }\end{array}$ \\
\hline TCP traffic & $\begin{array}{c}\text { Flows with audio code (G.711.2-84 Kbps and 50 pkts/s) transmitted } \\
\text { using real time protocol and voice activity detection }\end{array}$ \\
\hline VoIP traffic &
\end{tabular}

It is worth mentioning that the described scenario was constrained to a small number of end-user devices because our main objective was to show the feasibility of SIM-Know. We will perform more extensive evaluations on large emulated environments in our subsequent papers.

\subsection{Performance Metrics and Traffic Generation}

We compared SIM-Know to SSF and AHP-TOPSIS in terms of the number of handovers, number of throughput drops, handover latency, and various well-known network performance metrics (throughput, delay, jitter and packet loss) [42]. The quantity of handovers is the number of transfers an end-device makes when it moves from one place to another [43]. The throughput drops represent the times that the number of bytes transmitted falls to zero because of a handover [28]. The handover latency is the time that elapses between the instant that the user-device sends the last link-going-down message to the serving AP and the moment at which the end-user device establishes the connection with the target AP [3].

In the emulation experiments, scripts for generating traffic were developed by using the iPerf3 [44] and D-ITG [45] tools. We used D-ITG to generate VoIP flows with audio 
code (G.711.2 - $84 \mathrm{Kbps}$ and 50 pkts/s) transmitted using real-time protocol and voice activity detection. We used iperf3 to generate TCP flows with constant inter-departure time between packets (1000 pkts/s) and constant packet size (512 bytes).

\subsection{Results and Analysis}

Table 3 shows that SIM-Know and AHP-TOPSIS outperformed SSF, in terms of the number of handovers and the number of throughput drops, when the end-user device moved at any speed (slow, moderate, or high). This behavior is expected because SSF is the baseline and triggers a handover as soon as an AP with an RSSI higher than that of the serving AP is available.

Table 3. Handover Performance.

\begin{tabular}{cccc}
\hline Parameter & SSF & AHP-TOPSIS & SIM-Know \\
\hline & SlowMobility & & \\
\hline Number of handovers & 7 & 3 & 3 \\
\hline Number of throughput drops & 5 & 3 & 3 \\
\hline ModerateMobility & & 3 \\
\hline Number of handovers & 7 & 3 & 3 \\
\hline Number of throughput drops & 5 & 3 & 3 \\
\hline Number of handovers & HighMobility & & 1 \\
\hline Number of throughput drops & 7 & 4 & \\
\hline
\end{tabular}

Table 3 also reveals that when the end-user device moved at slow and moderate speeds, SIM-Know behaved as AHP-TOPSIS does regarding the number of handovers and the number of throughput drops. Figure 7 shows that SIM-Know outperformed AHP-TOPSIS in these metrics when the end-user device moved at a high velocity. The outperformance regarding the number of handovers and number of throughput drops was due to SIMKnow making context-aware, cognitive and proactive handovers. Figure 8 corroborates the fact that SIM-Know carried out handovers before SSF and AHP-TOPSIS did.

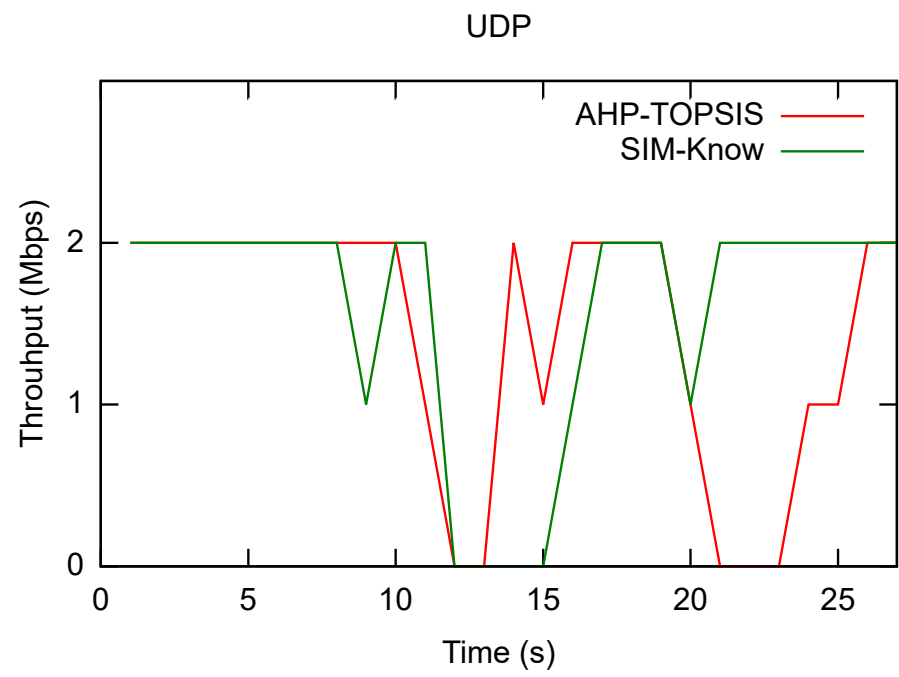

Figure 7. Throughput Drops.

Figure 9 shows, as expected, that SIM-Know obtained a higher handover latency than SSF did, since our approach is knowledge-based and SSF makes decisions considering a 
single criterion. SIM-Know had 27\% less handover latency than AHP-TOPSIS because, first, our approach is proactive and, according to [46], AHP-TOPSIS is reactive; the proactivity shortens the Handover Initiation phase [21]. Secondly, SIM-Know employs a rule-based reasoning method while AHP-TOPSIS uses a complex mathematical model that requires a considerable amount of time to make handover decisions.

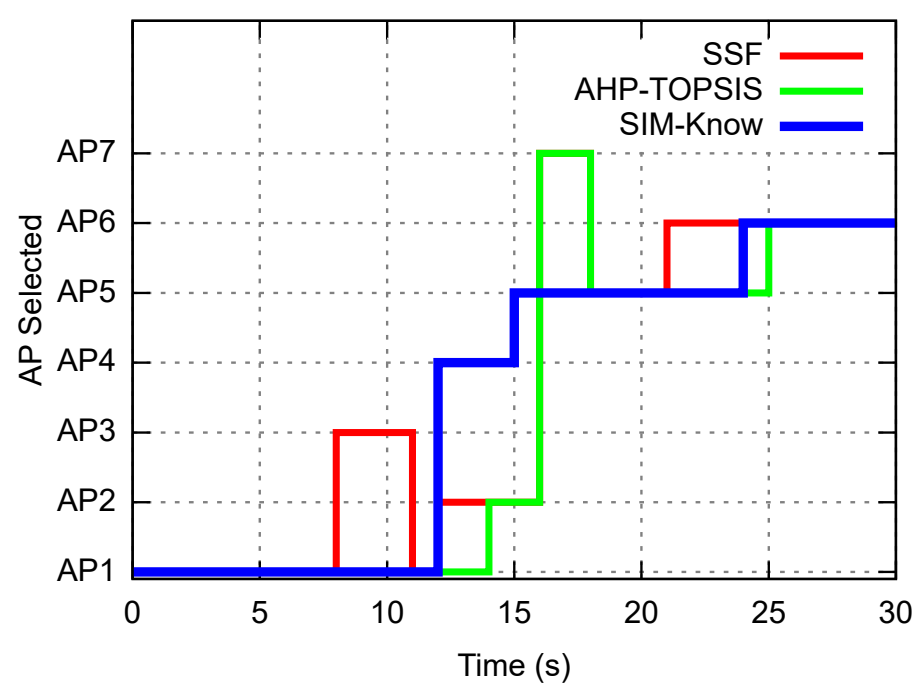

Figure 8. Proactivity.

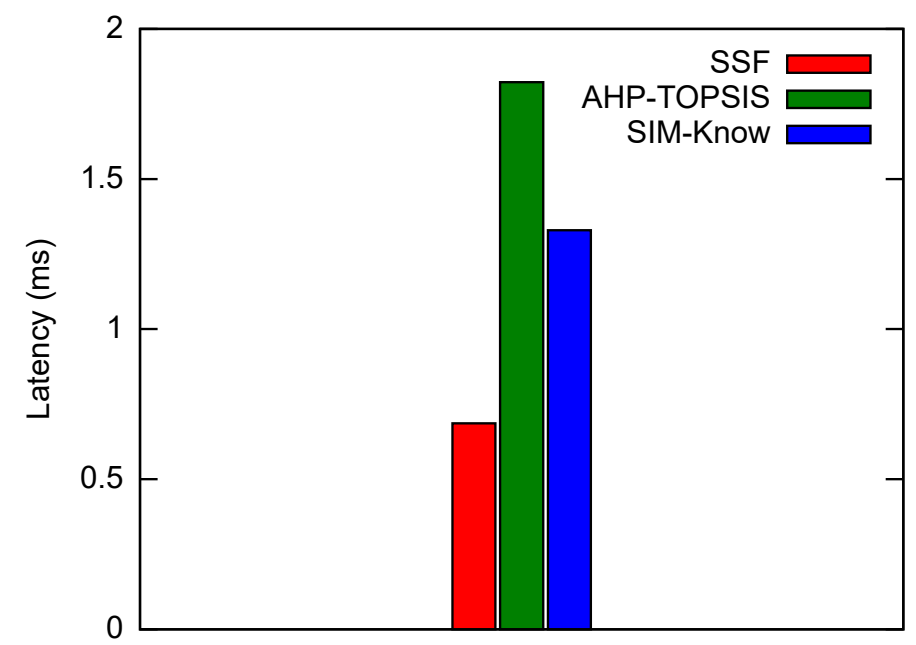

Figure 9. Handover Latency.

Next, we present how SIM-Know, SSF and AHP-TOPSIS impact various network performance metrics when the end-user device moves at HighMobility. Figure 10 depicts SIM-Know overcoming SSF and AHP-TOPSIS regarding the throughput, delay, and packet loss when the wireless network transferred VoIP/UDP traffic. In particular, the delay attained by SIM-Know was $29.28 \%$ and $23.13 \%$ lower than that achieved by SSF and AHPTOPSIS (see Figure 10a). The packet loss of SIM-Know was $99.44 \%$ and $98.38 \%$ lower than that obtained by SSF and AHP-TOPSIS (see Figure 10b). The throughput obtained by SIM-Know was $57.17 \%$ and $16.87 \%$ higher than that obtained by SSF and AHP-TOPSIS (see Figure 10c). Figure 11 shows that SIM-Know also outperformed SSF and AHP-TOPSIS regarding the throughput, delay and jitter when the wireless network transferred TCP traffic. Specifically, the delay attained by SIM-Know was $91.95 \%$ and $80.27 \%$ lower than that achieved by SSF and AHP-TOPSIS (see Figure 11a). The jitter performed by SIM-Know was $57.98 \%$ and $32.94 \%$ lower than that obtained by SSF and AHP-TOPSIS (see Figure 11b). The 
throughput accomplished by SIM-Know was $80.3 \%$ and $29.22 \%$ higher than that attained by SSF and AHP-TOPSIS (see Figure 11c).

We argue that the improvement in throughput, delay, jitter, and packet loss offered by SIM-Know is due to its context-aware, cognition and proactivity capabilities, which decreased the number of handovers and the number of throughput drops. In particular, SIM provides the context-aware capability to perform handover decisions through the comprehensive and semantic network view given by the information domains (Network, Application, User, UserDevice, and Handover). The KBP's Reasoning layer allows the achievement of cognitive HM. The distributed nature of KBP and its continuous updating allow the Handover Initiation phase to be proactive and operate with the local knowledge, built by $K B P_{M}$ and $K B P_{N}$, and the global knowledge, available in $K B P_{S}$. It is worth noting that the above results corroborate the idea that proactive approaches are more effective than reactive ones for meeting QoS requirements. On the other hand, we consider that the handover latency of SIM-Know can be addressed by improving the data format and the communication model between $K B P_{S}$ and both $K B P_{M}$ and $K B P_{N}$. These improvements are out of the scope of this paper and constitute limitations of the current SIM-Know version.

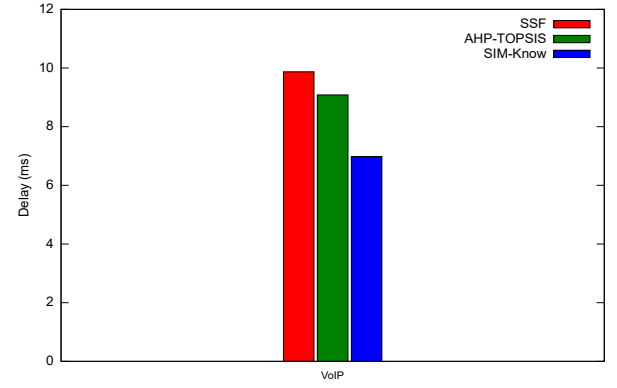

(a) Delay in VoIP Traffic at HighMobility

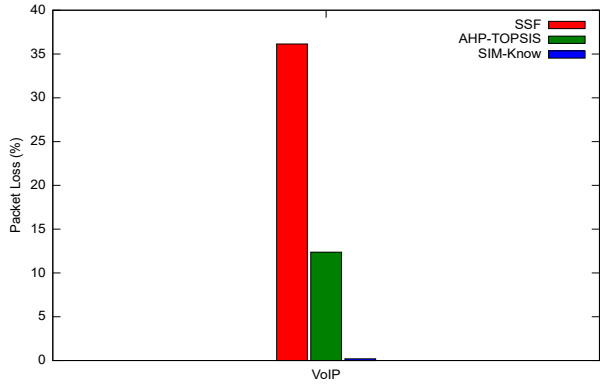

(b) Packet Loss in VoIP Traffic at HighMobility

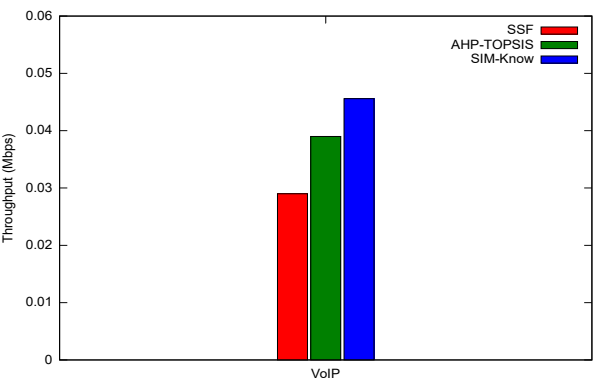

(c) Throughput in VoIP Traffic at HighMobility

Figure 10. Impact on VoIP Traffic.

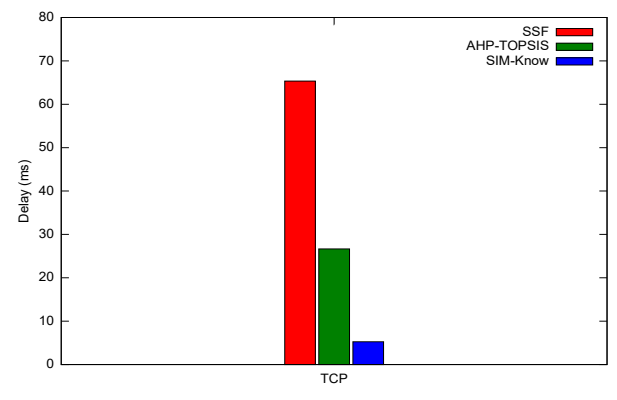

(a) Delay in TCP Traffic at HighMobility

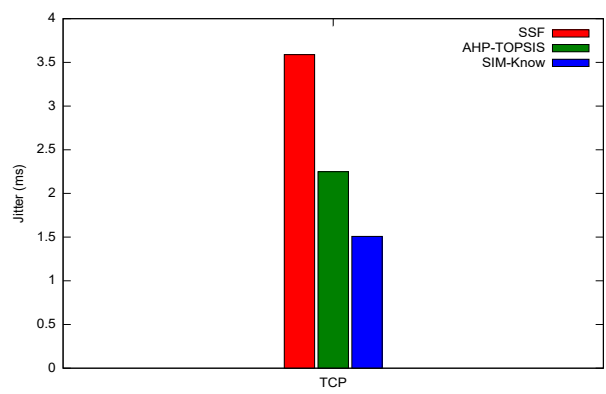

(b) Jitter in TCP Traffic at HighMobility

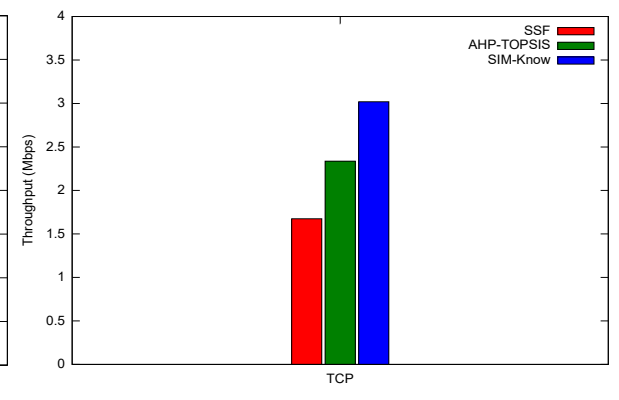

(c) Throughput in TCP Traffic at HighMobility

Figure 11. Impact on TCP Traffic.

\section{Conclusions and Future Work}

This paper introduced SIM-Know, an approach that performs context-aware, cognitive, and proactive handovers. SIM-Know includes SIM to provide context-awareness to handover decisions and KBP to incorporate cognition in HM. KBP distributes knowledge (local and global) to afford the proactivity capability in HM. The evaluation results showed that, thanks to the aforementioned SIM-Know capabilities, our approach overcomes SSF regarding the number of handovers and the number of throughput drops when the end-user device moves at any speed and, further, equals AHP-TOPSIS when it travels at low and moderate speeds. SSF outperforms SIM-Know and AHP-TOPSIS regarding the handover latency metric because SSF runs a straightforward process for making handover decisions. SIM-Know overcomes AHP-TOPSIS regarding all evaluated metrics when the end-user 
device moves at a high speed, positively impacting the wireless network's performance in terms of the delay, throughput, packet loss and jitter metrics. Considering these results, we concluded that SIM-Know is an attractive and feasible solution for cognitive HM.

For future work, we intend to enrich our approach with SDN and NFV capabilities to deal with the scalability issue imposed on HM by the Industrial IoT and the massive IoT $5 \mathrm{G}$ use case. We also plan to create an efficient model for communicating KBPs and evaluating SIM-Know when it makes handover decisions in scenarios with many end-user devices, high network traffic, and high load in APs.

Author Contributions: Conceptualization, F.Y.V., O.M.C. and J.C.N.; Investigation, F.Y.V., O.M.C. and J.C.N.; Methodology, F.Y.V., O.M.C. and J.C.N.; Writing—original draft, F.Y.V. and O.M.C.; Writingreview \& editing, F.Y.V., O.M.C. and J.C.N. All authors have read and agreed to the published version of the manuscript.

Funding: This work was supported by the InnovAccion Cauca project of the Colombian Science, Technology, and Innovation Fund (SGR-CTI-ID-3848) throuhg the PhD scholarship of the student Fulvio Vivas (01-2016).

Institutional Review Board Statement: Not applicable.

Informed Consent Statement: Not applicable.

Data Availability Statement: The SIM-Know Project Repository. 2021. Available online: https: // github.com/fyvivas/SIMKnow (accessed on 11 June 2021).

Acknowledgments: The authors would like to thank the Universidad del Cauca and the Umeå University.

Conflicts of Interest: The authors declare no conflict of interest. The financiers had no role in the design of the study; in the collection, analyses, or interpretation of data; in the writing of the manuscript, or in the decision to publish the results.

\section{Abbreviations}

The following abbreviations are used in this manuscript:

$\begin{array}{ll}\text { 3GPP } & \text { 3rd Generation Partnership Project } \\ \text { 5G } & \text { Fifth Generation } \\ \text { AHP } & \text { Analytic Hierarchy Process } \\ \text { AMF } & \text { Access and Mobility Management Function } \\ \text { AP } & \text { Access Point } \\ \text { BILP } & \text { Binary Integer Linear Programming } \\ \text { BS } & \text { Base Station } \\ \text { CIM } & \text { Common Information Model } \\ \text { CN } & \text { Core Network } \\ \text { DL } & \text { Description Logic } \\ \text { EN } & \text { Entity Notation } \\ \text { gNB } & \text { gNodeB } \\ \text { HF } & \text { Handover Failure } \\ \text { HM } & \text { Handover Management } \\ \text { IoT } & \text { Internet of things } \\ \text { KBP } & \text { Knowledge Base Profile } \\ \text { LTE } & \text { Long-Term Evolution } \\ \text { MADM } & \text { Multiple Attribute Decision Making } \\ \text { MAHO } & \text { Mobile-Assisted Handover } \\ \text { MCHO } & \text { Mobile-Controlled Handover } \\ \text { NAHO } & \text { Network-Assisted Handover } \\ \text { NCHO } & \text { Network-Controlled Handover } \\ \text { NFV } & \text { Network Function Virtualization } \\ \text { OWL } & \text { Web Ontology Language } \\ & \end{array}$




$\begin{array}{ll}\text { PP } & \text { Ping-Pong } \\ \text { PROMETHEE } & \text { Preference Ranking Organization METHod for Enrichment Evaluation } \\ \text { QoS } & \text { Quality of Service } \\ \text { RAN } & \text { Radio Access Network } \\ \text { RLF } & \text { Radio Link Failure } \\ \text { RSSI } & \text { Received Signal Strength Indication } \\ \text { SAW } & \text { Simple Additive Weighting } \\ \text { SDN } & \text { Software-Defined Networking } \\ \text { SIM } & \text { Semantic Information Model } \\ \text { SSF } & \text { Signal Strong First } \\ \text { TCP } & \text { Transmission Control Protocol } \\ \text { TOPSIS } & \text { Technique for Order Preferences by Similarity to the Ideal Solution } \\ \text { UDP } & \text { User Datagram Protocol } \\ \text { UE } & \text { User Equipment } \\ \text { UPF } & \text { User Plane Function } \\ \text { URLLC } & \text { Ultra-Reliable and Low Latency Communications } \\ \text { VM } & \text { Virtual Machine } \\ \text { VoIP } & \text { Voice over IP } \\ \text { WLAN } & \text { Wireless Local Area Network }\end{array}$

\section{References}

1. Ghosh, S.K.; Ghosh, S.C. A predictive handoff mechanism for 5G ultra dense networks. In Proceedings of the 2017 IEEE 16 th International Symposium on Network Computing and Applications (NCA), Cambridge, MA, USA, 30 October-1 November 2017; IEEE: New York, NY, USA, 2017; pp. 1-5. [CrossRef]

2. Calabuig, D.; Barmpounakis, S.; Gimenez, S.; Kousaridas, A.; Lakshmana, T.R.; Lorca, J.; Lunden, P.; Ren, Z.; Sroka, P.; Ternon, E.; et al. Resource and Mobility Management in the Network Layer of 5G Cellular Ultra-Dense Networks. IEEE Commun. Mag. 2017, 55, 162-169. [CrossRef]

3. Tayyab, M.; Gelabert, X.; Jantti, R. A Survey on Handover Management: From LTE to NR. IEEE Access 2019, 7, 118907-118930. [CrossRef]

4. Stamou, A.; Dimitriou, N.; Kontovasilis, K.; Papavassiliou, S. Autonomic Handover Management for Heterogeneous Networks in a Future Internet Context: A Survey. IEEE Commun. Surv. Tutor. 2019, 21, 3274-3297. [CrossRef]

5. Barmpounakis, S.; Kaloxylos, A.; Spapis, P.; Alonistioti, N. Context-aware, user-driven, network-controlled RAT selection for 5G networks. Comput. Netw. 2017, 113, 124-147. [CrossRef]

6. Akkari, N.; Dimitriou, N. Mobility management solutions for 5G networks: Architecture and services. Comput. Netw. 2020, 169, 107082. [CrossRef]

7. Bilen, T.; Canberk, B.; Chowdhury, K.R. Handover Management in Software-Defined Ultra-Dense 5G Networks. IEEE Netw. 2017, 31, 49-55. [CrossRef]

8. Zaidi, S.M.A.; Manalastas, M.; Farooq, H.; Imran, A. Mobility Management in Emerging Ultra-Dense Cellular Networks: A Survey, Outlook, and Future Research Directions. IEEE Access 2020, 8, 183505-183533. [CrossRef]

9. Jain, A.; Tokekar, S. Application Based Vertical Handoff Decision in Heterogeneous Network. In Procedia Computer Science; Elsevier: Amsterdam, The Netherlands, 2015; Volume 57, pp. 782-788. [CrossRef]

10. Khan, M.; Din, S.; Gohar, M.; Ahmad, A.; Cuomo, S.; Piccialli, F.; Jeon, G. Enabling multimedia aware vertical handover Management in Internet of Things based heterogeneous wireless networks. Multimed. Tools Appl. 2017, 76, 25919-25941. [CrossRef]

11. Din, S.; Ahmad, A.; Paul, A.; Rho, S. MGR: Multi-parameter Green Reliable communication for Internet of Things in 5G network. J. Parallel Distrib. Comput. 2018, 118, 34-45. [CrossRef]

12. Malathy, E.M.; Muthuswamy, V. State of Art: Vertical Handover Decision Schemes in Next-Generation Wireless Network. J. Commun. Inf. Netw. 2018, 3, 43-52. [CrossRef]

13. Zhao, P.; Yang, X.; Yu, W.; Lin, J.; Meng, D. Context-Aware Multi-Criteria Handover with Fuzzy Inference in Software Defined 5G HetNets. In Proceedings of the 2018 IEEE International Conference on Communications (ICC), Kansas City, MO, USA, 20-24 May 2018; IEEE: New York, NY, USA, 2018; pp. 1-6. [CrossRef]

14. Gaur, G.; Velmurugan, T.; Prakasam, P.; Nandakumar, S. Application specific thresholding scheme for handover reduction in 5G Ultra Dense Networks. Telecommun. Syst. 2021, 76, 97-113. [CrossRef]

15. Wang, X.; Qu, D.; Li, K.; Cheng, H.; Das, S.K.; Huang, M.; Wang, R.; Chen, S. A flexible and generalized framework for access network selection in heterogeneous wireless networks. Pervasive Mob. Comput. 2017, 40, 556-576. [CrossRef]

16. Hegazy, R.D.; Nasr, O.A.; Kamal, H.A. Optimization of user behavior based handover using fuzzy Q-learning for LTE networks. Wirel. Netw. 2018, 24, 481-495. [CrossRef]

17. Tartarini, L.; Marotta, M.A.; Cerqueira, E.; Rochol, J.; Both, C.B.; Gerla, M.; Bellavista, P. Software-defined handover decision engine for heterogeneous cloud radio access networks. Comput. Commun. 2018, 115, 21-34. [CrossRef] 
18. Barmpounakis, S.; Kaloxylos, A.; Spapis, P.; Magdalinos, P.; Alonistioti, N.; Zhou, C. Data Analytics for 5G Networks: A Complete Framework for Network Access Selection and Traffic Steering. Int. J. Adv. Telecommun. 2018, 11, 101-114.

19. Habbal, A.; Goudar, S.I.; Hassan, S. A Context-aware Radio Access Technology selection mechanism in 5G mobile network for smart city applications. J. Netw. Comput. Appl. 2019, 135, 97-107. [CrossRef]

20. Monteiro, A.; Souto, E.; Pazzi, R.; Nogueira, M. Context-aware network selection in heterogeneous wireless networks. Comput. Commun. 2019, 135, 1-15. [CrossRef]

21. Stamou, A.; Dimitriou, N.; Kontovasilis, K.; Papavassiliou, S. Context-aware handover management for HetNets: Performance evaluation models and comparative assessment of alternative context acquisition strategies. Comput. Netw. 2020, 176, 107272. [CrossRef]

22. Modeas, I.; Kaloxylos, A.; Merakos, L.; Tsolkas, D. An adaptive and distributed network selection mechanism for $5 \mathrm{G}$ networks. Comput. Netw. 2021, 189, 107943. [CrossRef]

23. Khan, M.; Han, K. A Vertical Handover Management Scheme based on Decision Modelling in Heterogeneous Wireless Networks. IETE Tech. Rev. 2015, 32, 402-412. [CrossRef]

24. Sadik, M.; Akkari, N.; Aldabbagh, G. SDN-based handover scheme for multi-tier LTE/Femto and D2D networks. Comput. Netw. 2018, 142, 142-153. [CrossRef]

25. Zekri, M.; Jouaber, B.; Zeghlache, D. A review on mobility management and vertical handover solutions over heterogeneous wireless networks. Comput. Commun. 2012, 35, 2055-2068. [CrossRef]

26. Salih, Y.K.; See, O.H.; Ibrahim, R.W.; Yussof, S.; Iqbal, A. An overview of intelligent selection and prediction method in heterogeneous wireless networks. J. Cent. South Univ. 2014, 21, 3138-3154. [CrossRef]

27. Han, Z.; Lei, T.; Lu, Z.; Wen, X.; Zheng, W.; Guo, L. Artificial Intelligence-Based Handoff Management for Dense WLANs: A Deep Reinforcement Learning Approach. IEEE Access 2019, 7, 31688-31701. [CrossRef]

28. Embus, D.A.; Castillo, A.J.; Vivas, F.Y.; Caicedo, O.M.; Ordóñez, A. NetSel-RF: A Model for Network Selection Based on Multi-Criteria and Supervised Learning. Appl. Sci. 2020, 10, 4382. [CrossRef]

29. Distributed Management Task Force, Inc. The CIM Standard: Common Information Model. 2021. Available online: https: / / www.dmtf.org/standards/cim (accessed on 25 April 2012).

30. Estrada-Solano, F.; Ordonez, A.; Granville, L.Z.; Caicedo Rendon, O.M. A framework for SDN integrated management based on a CIM model and a vertical management plane. Comput. Commun. 2017, 102, 150-164. [CrossRef]

31. W3C Recommendation. OWL Web Ontology Language. 2004. Available online: https://www.w3.org/TR/owl-features/ (accessed on 25 April 2012).

32. Nieves, J.C.; Espinoza, A.; Penya, Y.K.; de Mues, M.O.; Peña, A. Intelligence distribution for data processing in smart grids: A semantic approach. Eng. Appl. Artif. Intell. 2013, 26, 1841-1853. [CrossRef]

33. Bettini, C.; Brdiczka, O.; Henricksen, K.; Indulska, J.; Nicklas, D.; Ranganathan, A.; Riboni, D. A survey of context modelling and reasoning techniques. Pervasive Mob. Comput. 2010, 6, 161-180. [CrossRef]

34. Uzun, A.; von Hoffen, M.; Küpper, A. Enabling Semantically Enriched Data Analytics by Leveraging Topology-Based Mobile Network Context Ontologies. In Proceedings of the 4th International Conference on Web Intelligence, Mining and Semantics (WIMS14), Thessaloniki, Greece, 2-4 June 2014; Association for Computing Machinery: New York, NY, USA, 2014. [CrossRef]

35. Baader, F.; Calvanese, D.; McGuinness, D.L.; Nardi, D.; Patel-Schneider, P.F. The Description Logic Handbook; Cambridge University Press: Cambridge, UK, 2007. [CrossRef]

36. Silva, K.C.; Becvar, Z.; Cardoso, E.H.; Francěs, C.R. Self-tuning handover algorithm based on fuzzy logic in mobile networks with dense small cells. In Proceedings of the 2018 IEEE Wireless Communications and Networking Conference, WCNC, Barcelona, Spain, 15-18 April 2018; IEEE: New York, NY, USA, 2018; pp. 1-6. [CrossRef]

37. Su, X.; Riekki, J.; Haverinen, J. Entity Notation: Enabling knowledge representations for resource-constrained sensors. Pers. Ubiquitous Comput. 2012, 16, 819-834. [CrossRef]

38. European Telecommunications Standards Institute (ETSI). 5G; NR; NR and NG-RAN Overall Description; Stage-2. Technical Specification (TS) 138.300, 3rd Generation Partnership Project (3GPP). Version 16.4.0. Release 16. 2021. Available online: https:/ / www.etsi.org/deliver/etsi_ts/138300_138399/138300/16.04.00_60/ts_138300v160400p.pdf (accessed on 10 June 2021).

39. Fontes, R.R.; Afzal, S.; Brito, S.H.B.; Santos, M.A.S.; Rothenberg, C.E. Mininet-WiFi: Emulating software-defined wireless networks. In Proceedings of the Network and Service Management (CNSM), 2015 11th International Conference, Barcelona, Spain, 9-13 November 2015; IEEE: New York, NY, USA, 2015; pp. 384-389. [CrossRef]

40. Lantz, B.; Heller, B.; McKeown, N. A Network in a Laptop: Rapid Prototyping for Software-Defined Networks. In Proceedings of the 9th ACM SIGCOMM Workshop on Hot Topics in Networks, Monterey, CA, USA, 20-21 October 2010; Association for Computing Machinery: New York, NY, USA, 2010. [CrossRef]

41. SIM-Know Project Repository. 2021. Available online: https://github.com/fyvivas/SIMKnow (accessed on 11 June 2021).

42. Hanemann, A.; Liakopoulos, A.; Molina, M.; Swany, D.M. A study on network performance metrics and their composition. Campus-Wide Inf. Syst. 2006, 23, 268-282. [CrossRef]

43. Yu, H.W.; Zhang, B. A heterogeneous network selection algorithm based on network attribute and user preference. AD Hoc Netw. 2018, 72, 68-80. [CrossRef]

44. Dugan, J.; Elliott, S.; Mah, B.A.; Poskanzer, J.; Prabhu, K. iPerf3, Tool for Active Measurements of the Maximum Achievable Bandwidth on IP Networks. 2012. Available online: https:/ /iperf.fr/ (accessed on 25 April 2012). 
45. Avallone, S.; Guadagno, S.; Emma, D.; Pescape, A.; Ventre, G. D-ITG distributed Internet traffic generator. In Proceedings of the First International Conference on the Quantitative Evaluation of Systems, QEST 2004, Enschede, The Netherlands, 27-30 September 2004; IEEE: New York, NY, USA, 2004; pp. 316-317. [CrossRef]

46. Habbal, A.; Goudar, S.; Hassan, S. Context-aware Radio Access Technology Selection Approach in 5G Ultra Dense Networks. IEEE Access 2017, 5, 6636-6648. [CrossRef] 